\title{
A POWERFUL HYDRODYNAMIC BOOSTER FOR RELATIVISTIC JETS
}

\author{
Miguel A. Aloy ${ }^{1,2}$ and Luciano Rezzolla ${ }^{3,4,5}$ \\ Received 2005 November 17; accepted 2006 February 22; published 2006 March 14
}

\begin{abstract}
Velocities close to the speed of light are a robust observational property of the jets observed in microquasars and active galactic nuclei, and they are expected to be behind much of the phenomenology of gamma-ray bursts (GRBs). Yet the mechanism boosting relativistic jets to such large Lorentz factors is not fully known. Building on recent general relativistic, multidimensional simulations of progenitors of short GRBs, we discuss a new effect in relativistic hydrodynamics that can act as an efficient booster in jets. This effect is purely hydrodynamical and occurs when large velocities tangential to a discontinuity are present in the flow, yielding Lorentz factors $\Gamma \sim 10^{2}-10^{3}$ or larger in flows with moderate initial Lorentz factors. Although without a Newtonian counterpart, this effect can be explained easily through the most elementary hydrodynamical flow (i.e., a relativistic Riemann problem).
\end{abstract}

Subject headings: black hole physics — galaxies: jets — gamma rays: bursts — hydrodynamics — relativity — shock waves

\section{INTRODUCTION}

Determining the mechanism accelerating high-energy plasma to the relativistic velocities observed in jets from active galactic nuclei (AGNs) and microquasars, and expected in gamma-ray bursts (GRBs), remains a problem whose solution has long been sought in any consistent modeling of these astrophysical objects. Here we show how recent general relativistic simulations and novel effects of relativistic hydrodynamics can provide us with important clues in this search.

In crude but not oversimplified terms, the dynamics of a relativistic hydrodynamic jet in an external medium can be assimilated to the motion of two fluids, one of which is much hotter and at higher (or equal) pressure than the other one, and is moving with a large tangential velocity with respect to the cold, slowly moving fluid. In this scenario, a blob of hot plasma moving outward along the jet generically produces a hydrodynamical structure, in the direction perpendicular to the motion, composed of a "forward" shock (moving away from the jet axis) and of a "reverse" shock (moving toward the jet axis). This pattern of waves, which we indicate as $\mathcal{S C S}_{\rightarrow}$, where $\mathcal{S}$ refers to the reverse shock, $\mathcal{S} \rightarrow$ to the forward one, and $\mathcal{C}$ to the contact discontinuity between the two, is routinely produced in numerical simulations of relativistic jets. Surprisingly, however, because unexpected from Newtonian hydrodynamics, this pattern can change for sufficiently large tangential velocities, and in this case the inward-moving shock is replaced by a rarefaction wave, thus producing a $\mathcal{R C S}_{\rightarrow}$ pattern. Convincing evidence that this process is likely to occur under realistic conditions is now emerging from special relativistic calculations of extragalactic jets (Aloy et al. 2003) as well as from general relativistic, multidimensional simulations of progenitors of short GRBs (Aloy et al. 2005). The latter case is schematically shown in Figure 1, which shows the flow structure

\footnotetext{
${ }^{1}$ Max-Planck-Institut für Astrophysik, Karl-Schwarzschild-Strasse 1, 85741 Garching, Germany.

${ }^{2}$ Departamento de Astronomía y Astrofísica, Universidad de Valencia, C/ Dr. Moliner s/n, E-46100 Burjassot, Valencia, Spain.

${ }^{3}$ Max-Planck-Institut für Gravitationsphysik, Albert Einstein Institut, Am Muehlenberg 1, 14476 Golm, Germany.

${ }^{4}$ SISSA, International School for Advanced Studies and INFN, Via Beirut 2, I-34014 Trieste, Italy.

${ }^{5}$ Department of Physics and Astronomy, Louisiana State University, 202 Nicholson Hall, Baton Rouge, LA 70803.
}

produced in numerical simulations involving a thick accretion torus around a black hole and in which a ultrarelativistic outflow is generated.

Because disentangling this change in the wave pattern, first pointed out by Rezzolla \& Zanotti (2002), and understanding its influence on the complex dynamics of a relativistic jet can be very difficult, we resort to the simplest hydrodynamical flow in which this effect can occur (i.e., a Riemann problem).

\section{THE RELATIVISTIC BOOSTING MECHANISM}

We recall that a Riemann problem consists of determining the evolution of a fluid that is composed at some initial time by two uniform states (i.e., a "left" and a "right" state) with different and discontinuous hydrodynamical properties: the rest-mass density $\rho$, the pressure $p$, the specific internal energy $\epsilon$, the specific enthalpy $h \equiv 1+\epsilon / c^{2}+p /\left(\rho c^{2}\right)$, and the components of the velocity normal $v^{n}$ and tangential $v^{t}$ to the initial discontinuity. Using this initial setup and the equations of relativistic hydrodynamics, Rezzolla et al. (2003) revealed that, in contrast to what happens in Newtonian hydrodynamics, a smooth transition from one wave pattern to another can be produced by simply varying the velocities that are tangential to the initial discontinuity $v^{t}$, while maintaining the initial states unmodified. More specifically, for each set of initial states there exists a critical value for the initial tangential velocities at which a shock is transformed into a rarefaction wave or vice versa.

The discovery of this relativistic effect is not purely academic and is not without direct physical implications. Indeed, an important consequence of this change of waves is the possibility of producing a large amplification of the local Lorentz factor $\Gamma \equiv\left[1-\left(v^{t}\right)^{2}-\left(v^{n}\right)^{2}\right]^{-1 / 2}$ when, past a critical value for the tangential velocity, a shock wave transforms into a rarefaction wave. To illustrate how this can happen in practice, it is useful to consider a Riemann problem with initial states that would mimic the conditions for the generation of a GRB or the propagation of a plasma blob along a relativistic jet (either in a parsec-scale extragalactic source or in a microquasar). This can be done in practice by considering a left state that is much hotter, with larger pressure and smaller rest-mass density than the right state. Stated differently, this corresponds to the left state having a much larger specific enthalpy than the right one. Such a choice is justified by the fact that the jet is expected to be very underdense with respect to the external medium 


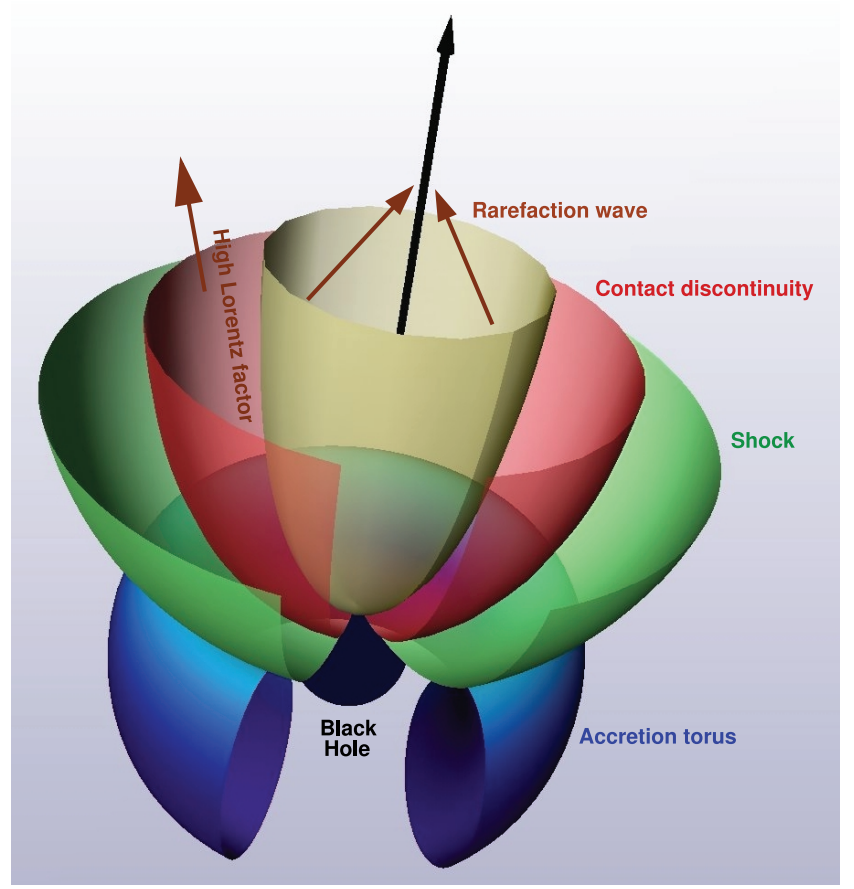

FIG. 1.-Schematic illustration of the flow structure in the acceleration of a short GRB produced by an accretion torus orbiting around a stellar mass black hole. The arrows mark the direction of fluid velocity at the rarefaction head (yellow paraboloidal surface), indicating that collimation of the fluid tends toward the black hole rotation axis (black arrow). A large boost is produced in the region between the rarefaction head and the contact discontinuity (red surface) separating the relativistic outflow from the shocked external medium.

(which could be given, for instance, by a dense accretion torus) and to be overpressured because a substantial change in the jet radius must happen between its launching site and the location at which it is detected $z$. For instance, one expects $z \approx$ $10^{13}-10^{14} \mathrm{~cm}$ for GRBs, while one expects $z \approx 10^{16}-10^{17} \mathrm{~cm}$ for microquasars and $z \approx 10^{18} \mathrm{~cm}$ for extragalactic jets.

Keeping these initial states fixed, we can then use the tangential velocity in the left state $v_{L}^{t}$ as a free parameter, whose tuning allows us to go from one wave pattern to the other. More specifically, let us consider the right state to be a cold fluid with a large rest-mass density and essentially at rest, i.e., with initial hydrodynamical properties: $p_{R} \approx 10^{-6} \rho_{\mathrm{ext}} c^{2}, \rho_{R} \approx 10^{-2} \rho_{\mathrm{ext}}, v_{R}^{n} \approx$ 0 , and $v_{R}^{t} \approx 0$, where $\rho_{\text {ext }}$ is a normalization constant that allows us to be scale-free. For convenience, hereafter we will assume $c=1$ and provide explicit values for $\rho_{\text {ext }}$ when discussing the astrophysical implications in $\S 3$. The left state will have comparable but larger pressure and will be underdense, with a small initial tangential velocity and a normal one close to the speed of light; i.e., $p_{L} \gtrsim \rho_{R}, \rho_{L} \ll \rho_{R}, v_{L}^{n} \approx 1$, and $v_{L}^{t} \approx 0$.

The solution of the Riemann problem produced by these two states after an arbitrary time is shown in the left panel of Figure 2 (we recall that the solution of the Riemann problem is self-similar; i.e., the structure of the solution in the different waves does not depend on time). The solution is of type $\mathcal{S C S}_{\rightarrow}$ and thus consists of a shock propagating to the left, of a shock propagating toward the right, and of a contact discontinuity at $x \approx 0.369$ separating the two waves. This doubleshock structure has a well-known Newtonian counterpart and is commonly observed in numerical simulations when there is almost pressure matching between the jet and the environment.
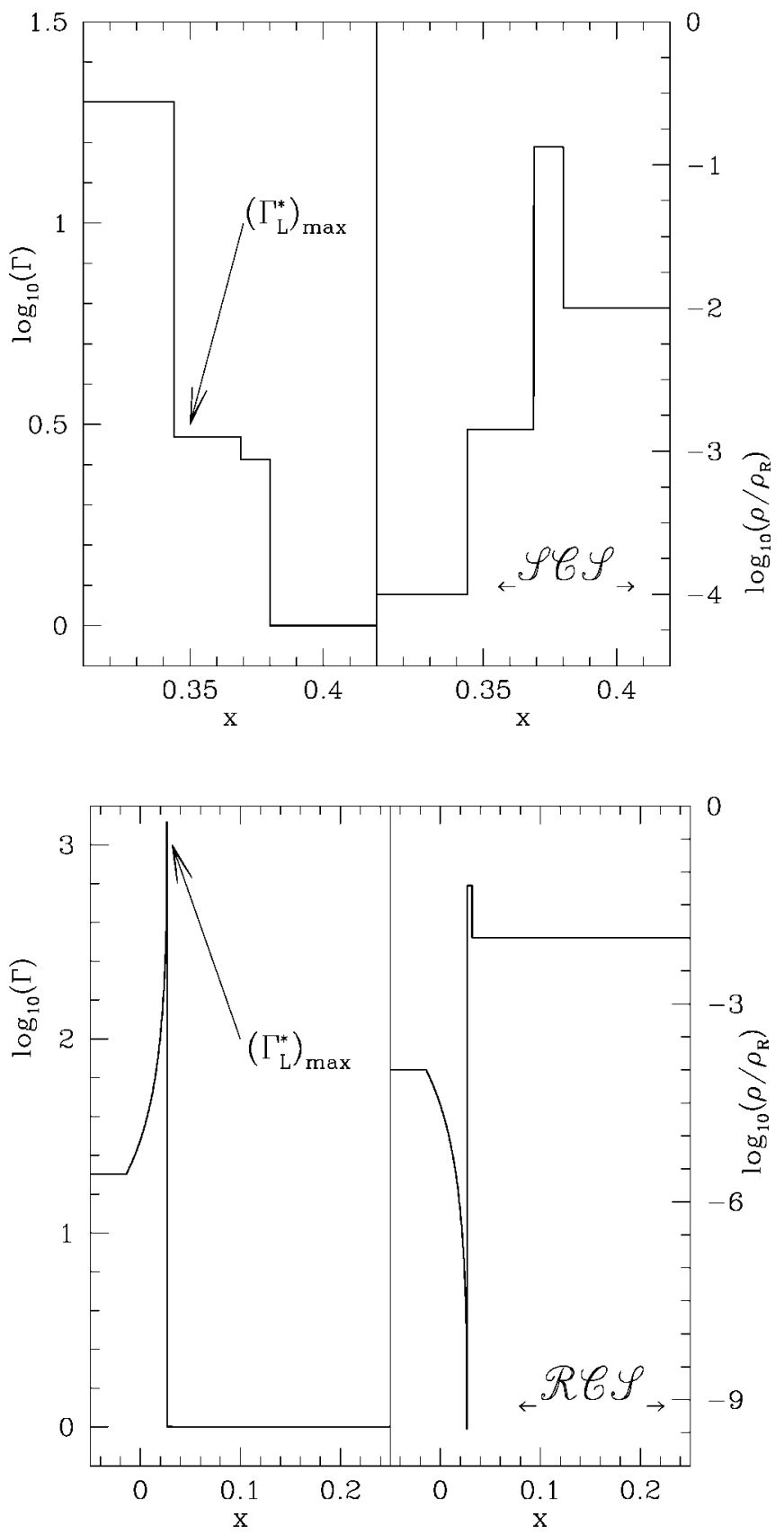

FIG. 2.-Left panel: Profiles for the Lorentz factor (left) and rest-mass density (right) resulting from a prototypical Riemann problem yielding a ${ }_{\triangle} \mathcal{S C S} \rightarrow$ pattern. The initial states are $\left(p_{L}, \rho_{L}, v_{L}^{n}, \Gamma_{L}\right)=\left(10^{-3}, 10^{-4}, 0.99,20\right)$ and $\left(p_{R}, \rho_{R}, v_{R}^{n}, \Gamma_{R}\right)=\left(10^{-6}, 10^{-2}, 0,1\right)$. Note that no boost is produced at the contact discontinuity [i.e., $\left(\Gamma_{L}^{*}\right)_{\max }<\Gamma_{L}$ ]. Right panel: Same as in the left panel, but for a prototype $\mathcal{R C S}_{\rightarrow}$ wave pattern. The initial states are $\left(p_{L}, \rho_{L}, v_{L}^{n}, \Gamma_{L}\right)=\left(10^{3}, 10^{-4}, 0,20\right) \quad$ and $\quad\left(p_{R}, \rho_{R}, v_{R}^{n}, \Gamma_{R}\right)=\left(10^{-6}, 10^{-2}, 0,1\right)$. Note in the left panel the very large boost [i.e., $\left(\Gamma_{L}^{*}\right)_{\max } \gg \Gamma_{L}$ ] that peaks at the contact discontinuity at $x \approx 0.027$.

Note that the acceleration at the transition layer between the jet and the external medium is very small and that the Lorentz factor reached in the state between the left-propagating shock and the contact discontinuity, $\Gamma_{L}^{*}$, is smaller than the initial Lorentz factor in the left state $\Gamma_{L}$. This is indicated as $\left(\Gamma_{L}^{*}\right)_{\max }$ in Figure 2 and reaches a value $\left(\Gamma_{L}^{*}\right)_{\max } \approx 2.943$ against an initial one in the left state $\Gamma_{L}=20$.

While keeping the right state to be the same, let us now consider the jet to be considerably overpressured and with almost 


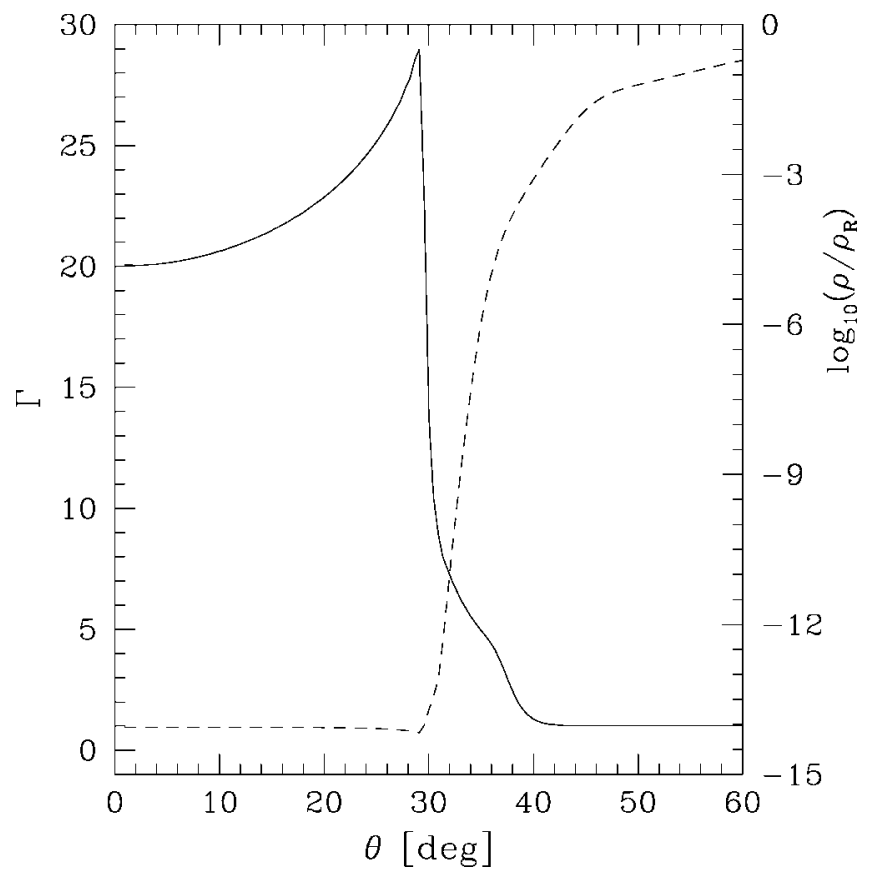

FIG. 3.-Example of the growth of the Lorentz from multidimensional simulations of ultrarelativistic jets generated in post-neutron star mergers (data from model A09 of Aloy et al. 2005). This is to be compared with the similar results shown in the right panel of Fig. 2.

no velocity component perpendicular to the jet direction. The left state can then be parameterized as $p_{L} \gg \rho_{R}, \rho_{L} \ll \rho_{R}, v_{L}^{n} \approx$ 0 , and $v_{L}^{t} \approx 1$, and the solution of the Riemann problem in this case is shown in the right panel of Figure 2. This is now of type $\leftarrow \mathcal{R C S} \rightarrow$ and consists of a rarefaction wave propagating to the left, of a contact discontinuity at $x \approx 0.027$, and of a shock propagating toward the right. This change in wave pattern is purely relativistic, and it is accompanied by a large increase in the Lorentz factor across the rarefaction wave, with the maximum value $\left(\Gamma_{L}^{*}\right)_{\max } \approx 10^{3}$ reached at the contact discontinuity.

This is a remarkable feature of relativistic hydrodynamics, namely, the production of a very strong rarefaction near the contact discontinuity and the combined conservation across such a wave of the specific enthalpy and of the Lorentz factor (i.e., of the quantity $h \Gamma$ ) have the consequence of accelerating the fluid to ultrarelativistic regimes with $\Gamma \sim 10^{4}$ or larger. In essence, this boosting mechanism efficiently converts into kinetic energy the work done on the jet by the external medium.

It is worth stressing again that the occurrence of these large fluid accelerations is not the result of peculiar initial conditions in a simplified hydrodynamical flow. Rather, accelerations produced through this effect are a robust feature in numerical simulations of relativistic jets (Aloy et al. 2003, 2005). In particular, the recent simulations of Aloy et al. (2005) show that, in spite of the complex multidimensional dynamics produced by the generation and propagation of the jet, the basic feature of this relativistic effect is preserved, i.e., the growth of the Lorentz factor close to the contact discontinuity separating a high-enthalpy and high-velocity state from a low-enthalpy one.

As an illustrative example, we report in Figure 3 results from an axisymmetric simulation in which the ultrarelativistic jet is overpressured with respect to the external medium and its velocity is almost parallel to the jet/external medium interface (Aloy et al. 2005). Shown along the polar $\theta$-direction at a distance of $3.2 \times 10^{7} \mathrm{~cm}$ from the black hole are the logarithm

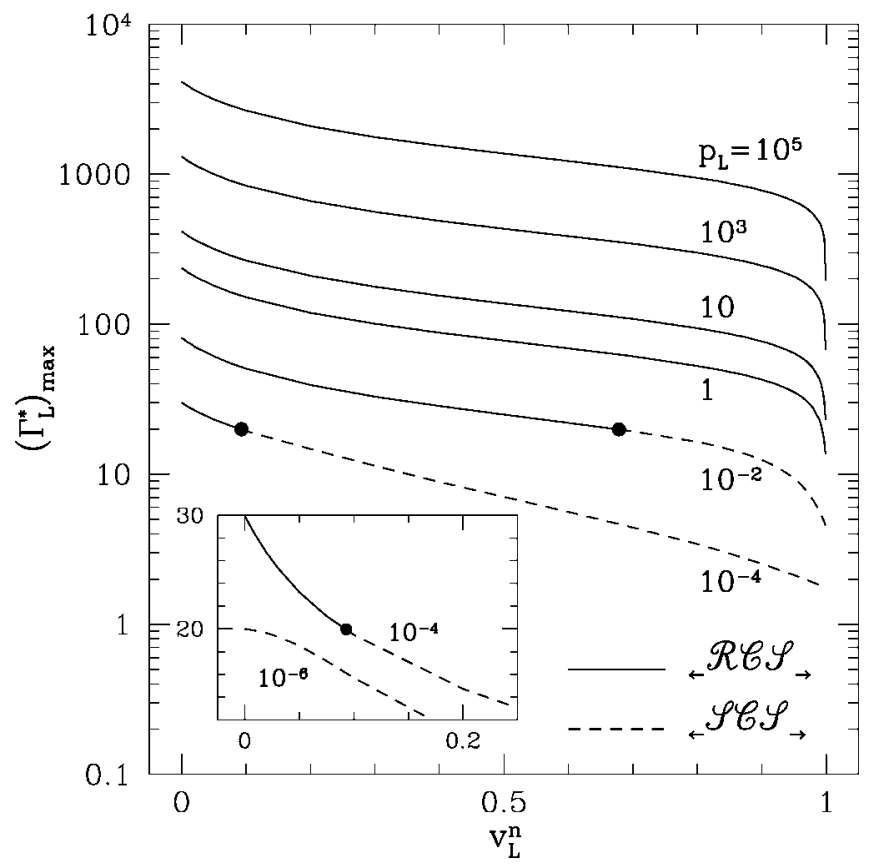

FIG. 4.-Maximum Lorentz produced in the Riemann problem $\left(\Gamma_{L}^{*}\right)_{\max }$ as a function of the normal velocity in the left state $v_{L}^{n}$. The right state is held fixed and is given by $\left(p_{R}, \rho_{R}, v_{R}^{n}, \Gamma_{R}\right)=\left(10^{-6}, 10^{-2}, 0,1\right)$. The left state has fixed values in the rest-mass density and a Lorentz factor $\left(\rho_{L}, \Gamma_{L}\right)=\left(10^{-4}, 20\right)$, while the pressure $p_{L}$ is varied for each curve as indicated by the different labels. The solid lines refer to a $\mathcal{R C S}_{\rightarrow}$ pattern, and the dashed lines refer to a $\mathcal{S C S}_{\rightarrow}$ pattern. The filled circles mark the critical normal velocity $\left(v_{L}^{n}\right)_{c}$.

of the rest-mass density (dashed line) and the profile of the Lorentz factor (solid line). The latter, in particular, reaches a maximum $\Gamma \approx 29$ in the left-going rarefaction wave moving toward the axis. These simulations have also revealed that although the interaction between the jet and the external medium is prone to Kelvin-Helmoltz and shear-driven instabilities (Aloy et al. 2002), the growth of the Lorentz factor imprinted during the initial phases of the jet propagation, i.e., when the jet interacts with the lateral borders of the accretion torus, remains preserved in the subsequent evolution of the flow.

Let us now illustrate in detail how the final fluid acceleration depends on the initial conditions by assuming that the right state is held fixed. In this way, in fact, the transition from one wave pattern to the other and the boost in the flow depend on two distinct but related factors: the value of the pressure in the left state $p_{L}$ and the value of the tangential velocity there, $v_{L}^{t}$.

In particular, it is possible to show that for very small leftstate pressures (i.e., $p_{L} \leqslant 10^{-6}$ for the initial states used above), the system evolves to produce a Riemann structure of the type $\mathcal{S C} \mathcal{S}_{\rightarrow}$ also when the normal velocity is zero (i.e., $v_{L}^{n} \approx 0$ ). On the other hand, for very large left-state pressures (i.e., $p_{L}>1$ for initial states used above), the system evolves to produce a Riemann structure of the type $\leftarrow \mathcal{R C S} \rightarrow$ also when the normal velocity is ultrarelativistic (i.e., $v_{L}^{n} \approx 1$ ). For all the values of $p_{L}$ within this range, a critical normal velocity $\left(v_{L}^{n}\right)_{c}$ exists below which the left-propagating shock is replaced by a rarefaction wave and a large boost takes place.

All of this is summarized in Figure 4, where the maximum values of the Lorentz factors are shown as a function of the initial value of the normal velocity in the left state $v_{L}^{n}$ (note that the value of $\Gamma_{L}$ is held fixed so that the increase of $v_{L}^{n}$ corresponds to a decrease of $v_{L}^{t}$ ). Different curves refer to different values 
of the pressure in the left state $p_{L}$, with solid lines indicating solutions of the Riemann problem of the type $\mathcal{R C S}_{\rightarrow}$, dashed lines to solutions of the type $\mathcal{S C S}_{\rightarrow}$, and filled circles marking the critical normal velocity $\left(v_{L}^{n}\right)_{c}$ at which one wave pattern transforms into the other.

\section{ASTROPHYSICAL IMPLICATIONS}

A first important astrophysical implication of the relativistic effects discussed here is that of requiring less extreme energy contents at the launching sites of relativistic jets and GRBs. This hydrodynamic mechanism, in fact, can boost a fluid to ultrarelativistic regimes very efficiently, and thus the Lorentz factors either expected or observed in astrophysical sources can be explained with initial "left states" that have considerably smaller pressures and are thus easier to produce in practice (in case the jet acceleration is mostly driven by pressure gradients).

To be more specific, for an ultrarelativistic jet expected in a short GRB, the conditions close to the generation site (i.e., above the poles of a rapidly rotating black hole) could be associated with a left state with $p_{L} \sim 10^{-2} \rho_{\mathrm{ext}}-10 \rho_{\mathrm{ext}}, \quad v_{L}^{n} \approx 0, \quad v_{L}^{t} \approx 1$, and $\rho_{L} / \rho_{R} \ll 1$ (for a short GRB progenitor, $\rho_{\text {ext }} \sim 1-10^{3} \mathrm{~g} \mathrm{~cm}^{-3}$; Janka et al. 1999). In this case, a Lorentz factor as small as $\Gamma_{L} \simeq 10$ is sufficient to produce, through the acceleration across the rarefaction wave, the inferred Lorentz factors of $\Gamma_{\text {obs }} \gtrsim 100$. Furthermore, because the pressure in the jet decreases more rapidly than the pressure in the external medium (i.e., $p_{L} \propto z^{-4}, p_{R} \propto z^{-3}$, where $z$ is the distance from the black hole), the boosting effect will be confined to a region within which $p_{L} / p_{R} \gtrsim 1$. For a black hole with a mass of $\sim 3 M_{\odot}$, this may happen for $z \lessgtr 300 \mathrm{~km}$. We also note that if the evolution of these boundary regions is maintained until the afterglow phase, then some differences in the emission properties are to be expected with respect to the standard model of the "universal" jet profile (i.e., a top-hat profile with sharp edges) or of the "structured-jet" model (i.e., where a progressive decay of the energy and of the Lorentz factor is expected from the jet core to the external medium). The impact of these structural changes within the jet on the light curve, whose calculation goes beyond the scope of this Letter, will help us to collect evidence on the occurrence of this relativistic acceleration.

In extragalactic relativistic jets, on the other hand, the Lorentz factors are more moderate, with $\Gamma_{\text {obs }} \lesssim 20$ (Vermeulen $\&$ Cohen 1994), and the physical conditions could then be associated with a left state with $p_{L} \sim 10^{-6} \rho_{\text {ext }}-10^{-4} \rho_{\text {ext }}, v_{L}^{n} \approx 0$, $v_{L}^{t} \approx 1$, and $\rho_{L} / \rho_{R} \sim 10^{-2}$ to $10^{-5}$ (for an extragalactic jet, $\rho_{\text {ext }} \sim 10^{-27}$ to $10^{-24} \mathrm{~g} \mathrm{~cm}^{-3}$; Ferrari 1998). In this case, a Lorentz factor $\Gamma_{L} \simeq 1.5$ is sufficient to produce the observed boosts. In addition, if a continuous channel of plasma is present together with the detected superluminal ejections, the physical conditions in galactic microquasar jets might be similar to the ones found in extragalactic jets, but with an even smaller inferred value of the Lorentz factor $\left(\Gamma_{\text {obs }} \lesssim 5\right.$; Mirabel \& Rodríguez 1999). In this case, values as small as $\Gamma_{L} \simeq 1.2$ would be sufficient to yield the observed apparent speeds.
A second important astrophysical implication of our results is of pointing out that, under suitable but realistic conditions, ultrarelativistic regimes can be reached very close to the jet launching site. Furthermore, while the boost originates and is larger in a small region at the interface between the jet and the external material, it is not restricted to a thin boundary layer but can rapidly involve the whole jet. This is because the leftgoing rarefaction wave can sweep up the jet material at relativistic speeds and thus rapidly accelerate a considerable mass fraction in the jet. As an example, on the basis of the values deduced from the Riemann problem considered in the right panel of Figure 2, more than $10 \%$ of the total (baryonic) mass is accelerated to $\Gamma>\Gamma_{L}$ in less that $\frac{1}{5}$ of the lateral jet-crossing time. In contrast, in other thermal acceleration mechanisms that also exploit the existence of $h \gg 1$ (e.g., the fireball model for GRBs), some time is needed to speed up the flow, with ultrarelativistic regimes being reached far from the launching site.

It is important to underline that the boosting mechanism proposed here should not be considered an alternative to the fireball model. Rather, it complements it by providing a more detailed description of the relativistic flows at the launching sites. Indeed, we expect both processes of hydrodynamic acceleration to operate simultaneously, with a longitudinal acceleration being produced by the expansion of a hot gas and a the lateral one being produced by the relativistic effects discussed here.

Interestingly, this boosting mechanism can also enhance particle acceleration when a turbulent velocity shear layer develops along a parsec-scale jet. These layers, in fact, whose existence has been pointed out both observationally (e.g., Owen et al. 1989; Swain et al. 1998) and through numerical simulations (Aloy et al. 1999, 2000), represent natural sites for particle acceleration, providing high-energy cosmic rays and influencing the dynamics of relativistic jets in extragalactic radio sources by forming cosmic-ray cocoons (Ostrowski 2000). The efficiency of the acceleration process in these turbulent shear layers depends on the particle mean free path and on the velocity structure (Stawarz \& Ostrowski 2002). Our results suggest that particle production can be further amplified if the simple flow structure usually assumed in these calculations and similar to the one produced in a $\mathcal{S C S}_{\rightarrow}$ is instead replaced by a more realistic one, such as the one occurring in a $\mathcal{R C S}_{\rightarrow}$.

As a final remark, we point out that the large hydrodynamic boosts reported here may be detected with laboratory experiments involving heavy-ion collisions. In these experiments, heavy ions are accelerated to ultrarelativistic velocities and collided. If the two beams are chosen to have different specific enthalpies and to collide with a nonzero impact parameter, an acceleration of the interacting layer could be produced as a result of the relativistic boosting discussed here.

M. A. A. is a Ramón y Cajal Fellow of the Spanish MEC. Support for this research also comes from the Spanish MEC (AYA2004-08067-C03-C01) and the SFB-TR7 "Gravitationswellenastronomie" of the DFG.

\section{REFERENCES}

Aloy, M. A., Gómez, J.-L., Ibáñez, J. M., Martí, J. M., \& Müller, E. 2000, ApJ, 528, L85

Aloy, M. A., Ibáñez, J. M., Martí, J. M., Gómez, J.-L., \& Müller, E. 1999, ApJ, 523, L125

Aloy, M. A., Ibáñez, J.-M., Miralles, J.-A., \& Urpin, V. 2002, A\&A, 396, 693

Aloy, M. A., Janka, H.-T., \& Müller, E. 2005, A\&A, 436, 273

Aloy, M. A., Martí, J., Gómez, J., Agudo, I., Müller, E., \& Ibáñez, J. 2003, ApJ, 585, L109

Ferrari A, 1998, ARA\&A, 36, 539
Janka, H.-T., Eberl, T., Ruffert, M., \& Fryer, C. L. 1999, ApJ, 527, L39

Mirabel, I. F., \& Rodríguez, L. F. 1999, ARA\&A, 37, 409

Ostrowski, M. 2000, MNRAS, 312, 579

Owen, F. N., Hardee, P. E., \& Cornwell, T. J. 1989, ApJ, 340, 698

Rezzolla, L., \& Zanotti, O. 2002, Phys. Rev. Lett., 89, 114501

Rezzolla, L., Zanotti, O., \& Pons, J. A. 2003, J. Fluid Mech., 479, 199

Stawarz, Ł., \& Ostrowski, M. 2002, ApJ, 578, 763

Swain, M. R., Bridle, A. H., \& Baum, S. A. 1998, ApJ, 507, L29

Vermeulen, R. C., \& Cohen, M. H. 1994, ApJ, 430, 467 